http://jmscr.igmpublication.org/home/ ISSN (e)-2347-176x ISSN (p) 2455-0450 crossref DOI: https://dx.doi.org/10.18535/jmscr/v8i6.51

\title{
Ultrasound Guided Preoperative Airway Assessment and Its Correlation with Standard Clinical Airway Assessment in Patients Having BMI $>23 K G / M^{2}$
}

\author{
Authors \\ Dr Kanika Sharma ${ }^{1}$, Dr Kartik Syal ${ }^{2}$, Dr Rajesh Verma ${ }^{3}$, Dr Surinder Singh ${ }^{4}$ \\ ${ }^{1}$ Medical Officer Specialist (Anesthesia) at DDUZH Shimla H.P \\ ${ }^{2}$ Associate Professor Dept. of Anesthesia at IGMC Shimla H.P \\ ${ }^{3}$ Assistant professor Dept. of Anaesthsia at IGMC Shimla H.P \\ ${ }^{4}$ Professor and Head Dept. of Anesthesia at IGMC Shimla H.P
}

\section{Introduction}

Successful airway management requires a range of knowledge and skills to predict difficulty and to formulate an airway management plan as well as to have the skills necessary to execute the plan using the wide array of airway devices available. Difficult laryngoscopy and tracheal intubation rate still remains at $1.5-13 \%$ due to poor reliability of traditional protocols, algorithms and combinations of screening tools in identifying a potentially difficult airway. ${ }^{1}$ Various studies had shown that the incidence of difficult bag and mask ventilation in overweight patients is approximately $6 \%$ whereas in lean patients is approximately $1 \%$ and incidence of difficult intubation in overweight is $1.5-16 \%$ and in lean it is approximately $7 \% .^{2}$

Clinical criterias routinely used to assess patients prior to anesthesia have variations according to patient's height, weight and ethnicity.

Ultrasonography (USG) is well-known as a fast, safe and noninvasive technique. There were very limited studies which showed value of USG derived parameters in assessing airway in obese patients and its co-relation with proven clinical parameters. The clinical parameters (MPS, TMD, neck circumference) which were included in the present study are the proven ones to assess airway, especially in overweight and obese patients. The USG derived parameters, preepiglotic space depth (PES), epiglottis to vocal cord distance (EVC), skin to dorsum of tongue distance (S-DT) are the USG parameters taken and clinically assessed parameters and USG parameters were co-related with each other taking Cormacke-Lehane grading as a reference point.

\section{Material and Methods}

Prospective and observational study was carried out on 100 patients in the age group of 18-60 years of either gender after getting approval for institutional ethical committee coming for surgery and having $\mathrm{BMI}>23 \mathrm{~kg} / \mathrm{m}^{2}$ (overweight and obese according to WHO BMI guidelines for Asian population) in the Department of Anesthesiology with sample size of 100 , having sensitivity of $80 \%$ and taking incidence of difficult intubation in overweight patients as $20 \%{ }^{3}$

\section{Exclusion Criteria}

Patient's refusal to participate in the study, Rapid sequence induction of anesthesia. 
Inability to open the mouth due to existing trauma or medical condition, Preexisting neck or facial disease,

Preexisting limitation or pain with cervical spine movement.

Clinical parameters (NC, TMD, MPS) were measured and USG parameters (PES, EVC, S-DT) were measures using a SonoSite ${ }^{\circledR}$ MicroMaxx ${ }^{\circledR}$ using linear and curvilinear probe.

All the patients were induced and intubated by a senior anaesthesiologist with more than 5 years of experience. Bag and mask ventilation and Direct laryngoscopy was done and BMV grade and CL grade was noted. BMV and CL grade was classified as easy (Grade 1 and 2) or difficult (Grade 3 and 4). Microsoft Excel spreadsheet cleaned it for errors and analyzed the data using Stata IC software version 15. Quantitative data is presented as mean $\pm \mathrm{SD}$. For comparison of means between two groups Independent t-test or Wilcoxen rank sum was used. For categorical variables, number \& percentages and their 95\% Confidence interval is calculated. Chisquare test was applied to see association between different categorical variables. To calculate optimal cut-off values of different variables ROC curve was used. Area under curve was used to compare diagnostic accuracy of different variables. For all variables sensitivity, specificity, Positive and negative predictive values were calculated at cutoff value as per ROC Curve. A two sided $P$ value of $<0.05$ was considered as statistical significant.

\section{Result}

Among 100 patients $83 \%$ were of BMV grade 1,2 and $17 \%$ were of BMV grade $3,4.73 \%$ were of CL grade 1, 2 and $27 \%$ were of CL grade 3,4.

\section{Mean Values of Parameters W.R.T BMV Grade}

\begin{tabular}{|l|c|c|c|}
\hline PARAMETER & EASY(G1+G2) & DIFFICULT(G3+G4) & p VALUE \\
\hline NC $($ mean \pm SD) & $35.8 \pm 2.3$ & $38.8 \pm 2.3$ & $<0.001$ \\
\hline TMD $($ mean \pm SD) & $6.5 \pm 0.7$ & $5.8 \pm 0.5$ & $<0.001$ \\
\hline PES $($ mean \pm SD) & $1.0 \pm 0.3$ & $1.3 \pm 0.4$ & $<0.001$ \\
\hline EVC $($ mean \pm SD) & $1.9 \pm 0.5$ & $1.9 \pm 0.5$ & 0.943 \\
\hline S-DT $($ mean \pm SD) & $5.5 \pm 0.6$ & $6.3 \pm 0.5$ & 0.001 \\
\hline
\end{tabular}

Mean Values of Parameters W.R.T CL Grade

\begin{tabular}{|l|c|c|c|}
\hline PARAMETER & EASY $($ G1+G2) & DIFFICULT(G3+G4) & p VALUE \\
\hline NC $($ mean \pm SD) & $35.8 \pm 2.2$ & $37.7 \pm 3.0$ & 0.001 \\
\hline TMD $($ mean \pm SD) & $6.5 \pm 0.8$ & $6.1 \pm 0.5$ & 0.001 \\
\hline PES $($ mean \pm SD) & $1.0 \pm 0.3$ & $1.2 \pm 0.4$ & 0.002 \\
\hline EVC $($ mean \pm SD) & $1.9 \pm 0.5$ & $1.9 \pm 0.4$ & 0.755 \\
\hline S-DT $($ mean \pm SD) & $5.6 \pm 0.6$ & $5.9 \pm 0.6$ & 0.058 \\
\hline
\end{tabular}

\section{Diagnostic Accuracy with BMV Grade}

\begin{tabular}{|l|c|c|c|c|c|c|}
\hline \multirow{2}{*}{} & \multicolumn{3}{|c|}{ CLINICAL } & \multicolumn{3}{c|}{ USG } \\
\cline { 2 - 7 } & NC & TMDA & MPS & P-ES & E-VC & S-DT \\
\hline SENSITIVITY & 100 & 94.1 & 29.4 & 94.1 & 52.9 & 94.1 \\
\hline SPECIFICITY & 54.2 & 62.7 & 72.3 & 56.6 & 51.8 & 61.4 \\
\hline PPV & 30.9 & 34.0 & 17.9 & 30.8 & 18.4 & 33.3 \\
\hline NPV & 100.0 & 98.1 & 83.3 & 97.9 & 84.3 & 98.1 \\
\hline Area under curve (Total) & 0.83 & 0.75 & 0.51 & 0.81 & 0.51 & 0.85 \\
\hline CUTOFF of ROC curve & $\geq 36.0$ & $\leq 6.2$ & $\geq 2.0$ & $\geq 1.0$ & $\geq 2.0$ & $\geq 5.8$ \\
\hline
\end{tabular}




\section{Diagnostic Accuracy with CL Grade}

\begin{tabular}{|c|c|c|c|c|c|c|}
\hline & \multicolumn{3}{|c|}{$\begin{array}{c}\text { CLINICAL } \\
\text { PARAMETERS }\end{array}$} & \multicolumn{3}{|c|}{$\begin{array}{c}\text { USG } \\
\text { PARAMETERS }\end{array}$} \\
\hline & $\mathrm{NC}$ & TMD & MPS & P-ES & E-VC & S-DT \\
\hline SENSITIVITY & 66.7 & 74.1 & 22.2 & 74.1 & 63.0 & 74.1 \\
\hline SPECIFICITY & 69.9 & 63.0 & 69.9 & 56.2 & 53.4 & 61.6 \\
\hline PPV & 45.0 & 42.6 & 21.4 & 38.5 & 33.3 & 41.7 \\
\hline NPV & 85.0 & 86.8 & 70.8 & 85.4 & 79.6 & 86.5 \\
\hline Area under curve & 0.70 & 0.67 & 0.46 & 0.70 & 0.54 & 0.66 \\
\hline CUTOFF of ROC curve & $\geq 37.0$ & $\leq 6.2$ & $\geq 2.0$ & $\geq 1.0$ & $\geq 1.93$ & $\geq 5.8$ \\
\hline
\end{tabular}

\section{Discussion}

There is a need of non-invasive methods which can predict $\mathrm{CL}$ grading and BMV grade preoperatively with a higher predictive value. The advent of ultrasonography has brought a paradigm shift in the practice of airway management.

Bag and mask ventilation: Among all the patients, patients with easy BMV i.e. grade 1or 2 were $83 \%$ and with difficult BMV i.e. GRADE 3 or 4 were 17\%. Mean age and BMI for easy BMV grade $(\mathrm{G} 1+\mathrm{G} 2)$ was $45.3 \pm 13.2 \mathrm{yr}$ and $25.8 \pm 1.5 \mathrm{~kg} / \mathrm{m}^{2}$ and for difficult BMV grade $(\mathrm{G} 3+\mathrm{G} 4)$ was $47.5 \pm$ $12.0 \mathrm{yr}$ and $27.7 \pm 3.8 \mathrm{~kg} / \mathrm{m}^{2}$ respectively.

Neck circumference (NC) is the best parameter among the clinical parameters with highest diagnostic accuracy i.e. 0.83, skin to dorsum of tongue distance (S-DT) is the best parameter among USG parameters with diagnostic accuracy i.e. 0.85 for anticipating difficult BMV.

Lee and co-workers ${ }^{4}$ did a study on 110 consecutive adult patients (66 men and 44 women). The age of the patients ranged from 21 to 96 years and incidence of difficult BMV was encountered in 51 patients $(45.9 \%)$. Mean of TMD for difficult BMV was found $6.6 \pm 1.2 \mathrm{~cm}$ thus coinciding with our findings. Slight difference value of TMD in predicting difficult BMV may be due to difference in criteria for estimating difficult BMV.

Cormack lehane grade: The patients were classified according to CL grading system. Easy laryngoscopy was defined as CL grade 1, 2 and difficult laryngoscopy was defined as CL grade 3, 4. In present study among total 100 patients number of patients having easy intubation i.e. CL grade 1,2 were $73 \%$ and with difficult intubation i.e. CL grade 3,4 were $27 \%$.

Neck circumference (NC) is the best parameter among the clinical parameters with diagnostic accuracy i.e. 0.70, preepiglottic space depth (PES) depth among USG parameters with diagnostic accuracy i.e. 0.70 for anticipating difficult intubation.

Ittiara et $a l^{5}$ in 2011 studied 50 cases. They observed a correlation of the distance between the epiglottis and the vocal cords (E-VC) with the Cormack Lehane Grading. The correlation of the pre-epiglottis space (PES) with the Cormack Lehane Grading was strong in positive direction. Which is similar to our study i.e. PES depth has highest diagnostic accuracy.

\section{Summary \& Conclusion}

Thus we summarise from our study that USG is a useful aid in airway assessment for predicting difficult airway in perioperative settings.

Thus USG being noninvasive and easily available equipment we recommend its further use in airway assessment, though more studies are needed to clearly define its role and protocols for the same. In the present scenario it is at best suggested that USG is an additive aid to clinical tests for predicting difficult airway of patient.

\section{References}

1. Khan ZH, Kashfi A, Ebrahimkhani E. A comparison of the upper lip bite test (a simple new technique) with modified Mallampati classification in predicting difficulty in endotracheal intubation: a 
prospective blinded study. Anesth Analg 2003; 96(2):595-9.

2. Shailaja S, Nichelle SM, Shetty AK, Hegde BR. Comparing ease of intubation in obese and lean patients using intubation difficulty scale. Anesth Essays Res 2014; 8: 168-74.

3. Ezri T, Gewurtz G, Sessler DI, Medalion B, Szmuk P, Hagberg C. Prediction of difficult laryngoscopy in obese patients by ultrasound quantification of anterior neck soft tissue. Anaesthesia2003; 58: 1111-14

4. Lee SY, Chien DK, Huang MY, Huang $\mathrm{CH}$, Shih SC, Wu KM, Ku HC, Chang WH. Patient specific factors associated with difficult mask ventilation in emergency department. IJGE 2017; 11(4): 263-6.

5. Ittiara B, Gupta D, Apple L, Srirajakalidindi A, Toshniwal G, Haber H. Ultrasonographic modification of Cormack Lehane classification for pre-anesthetic airway assessment. Middle East $\mathbf{J}$ Anesthesiol2012; 21(6): 835-42. 